Reporte de caso

\title{
Estudio patológico de abiotrofia cerebelosa en American Staffordshire terrier: primer reporte en Uruguay
}

\section{Pathological study of cerebellar abiotrophy in American Staffordshire terrier: first report in Uruguay}

José Manuel Verdes ${ }^{1,4^{*}} 0000-0003-4314-906 X$

Luis Delucchi² 0000-0001-9659-228X

Gimena Feijoó ${ }^{1,2,3}$ 0000-0002-1729-2316
Fernando Fumagalli',5 0000-0001-9127-3546

Carlos Rodríguez Serpa² 0000-0002-3373-0195

Kanji Yamasaki ${ }^{1}$ 0000-0001-5175-0053

${ }^{1}$ Área Patología, Departamento de Patología, Facultad de Veterinaria, UdelaR. Av. Alberto Lasplaces 1550-1620, CP 11600,

Montevideo, Uruguay. * Email: jmverdes@fvet.edu.uy

${ }^{2}$ Unidad de Neurología, Departamento de Patología y Clínica de Pequeños Animales, FVET-UdelaR.

${ }^{3}$ Área Farmacología, Departamento de Fisiología, FVET-UdelaR.

${ }^{4}$ Área Biofísica, Departamento de Biología Molecular y Celular, FVET-UdelaR.

${ }^{5}$ Área Semiología, Centro Hospital Veterinario, FVET-UdelaR.

Veterinaria (Montevideo) Volumen 56

№ 213 (2020 Ene - Jun) e20205621304

\section{Resumen}

Se observó abiotrofia cerebelosa en una hembra American Staffordshire terrier de 6 años. La perra mostraba temblores, debilidad y ataxia como signos clínicos principales al momento de la consulta al Centro Hospital Veterinaria (FVET-UdelaR), y estos síntomas siguieron progresando lentamente durante 6 meses. El animal fue sometido a eutanasia debido a la solicitud de los propietarios, ya que no había posibilidad de recuperación, y se realizó su examen patológico. Macroscópicamente, el cerebelo estaba pequeño y sus folias estaban adelgazadas y con expansión de los surcos interfoliares. Histológicamente, las tres capas de la corteza cerebelosa mostraban una estructura anormal, caracterizada por una disminución en el número de células en la capa granulosa, pérdida de células de Purkinje y adelgazamiento de la capa molecular. A partir de estos hallazgos patológicos, este caso fue diagnosticado como abiotrofia cerebelosa canina. Por lo que sabemos, este es el primer caso reportado de esta enfermedad en Uruguay.

Palabras clave: Abiotrofia, Cerebelo, Diagnóstico patológico, Canino.
Recibido: : 22/06/2019

Aceptado: 18/03/2020 $(c))$ EY-NC

\section{Abstract}

Cerebellar abiotrophy was observed in a female American Staffordshire terrier aged 6 years. The bitch showed tremors, weakness, ataxia as main clinical signs at the time of visit the Veterinary Hospital (FVET-UdelaR), and these signs were progressed slowly for 6 months. The dog was euthanatized due to owners offer, considering no possibility of recovery, and was examined pathologically. Grossly, the cerebellum was small, and the folia were thin, with expanded interfoliar sulci. Histologically, the three layers of the cerebellar cortex showed abnormal structure, characterized by decrease in number of cells in the granular layer, loss of Purkinje cells and thinning of molecular layer. From these pathological findings, this case was diagnosed as canine cerebellar abiotrophy. To our knowledge, this is the first case report of this disease in Uruguay.

Keywords: Abiotrophy, Cerebellum, Diagnostic pathology, Dog. 


\section{Introducción}

La abiotrofia cerebelosa es una degeneración cortical progresiva e irreversible que se manifiesta desde algunos meses hasta años luego del nacimiento en varias razas caninas, causada por un trastorno metabólico hereditario. La misma condición se ha descrito en otras especies, como bovinos, ovinos, equinos, suinos, caprinos, felinos y también en humanos (de Lahunta, 1990; Scott et al., 2018). En perros, esta enfermedad ha sido reportada desde hace tiempo en varias razas, incluyendo Collie (Gill y Hewland, 1980), Kelpie australiano (Thomas y Robertson, 1989), Labrador retriever (Perille et al., 1991), Rhodesian ridgeback (Chieffo et al., 1994), Boyero de Berna (Carmichael et al., 1996), Beagle (Kent et al., 2000), Podenco portugués (van Tongeren et al., 2000), Scottish terrier (van der Merwe y Lane, 2001), Schnauzer miniatura (Berry y Blas-Machado, 2003), Bulldog inglés (Gandini et al., 2005), Lagotto romagnolo (Jokinen et al., 2007) y Staffordshire terrier (Speciale y de Lahunta, 2003), mientras que también se han reportado abiotrofias cerebelosas familiares de inicio tardío en Bretón (Yasuba et al.,1988), Rhodesian ridgeback (Chieffo et al., 1994), Old English sheepdog (Steinberg et al., 2000; Agler et al., 2014), Border collie (Sandy et al., 2002), Kelpie Australiano (Shearman et al., 2011), Scottish terrier (Urkasemsin et al., 2010; 2017), Golden setter (Agler et al., 2014) y American Staffordshire terrier (Olby et al., 2004, Siso et al., 2004, Henke et al.,2008, Abitbol et al.,2010). Aunque se ha investigado la utilidad de la imagenología por resonancia magnética para el diagnóstico de esta enfermedad (Henke et al., 2008), aún se considera que el diagnóstico de esta enfermedad es difícil solo en base a los hallazgos clínicos, siendo el diagnóstico patológico fundamental para arribar al diagnóstico definitivo. En este trabajo se comunican los hallazgos de necropsia e histopatología encontrados en una perra American Staffordshire terrier que mostraba signos neurológicos, y a la que se le diagnosticó por estudio patológico completo, abiotrofia cortical cerebelosa. Por lo que sabemos, este es el primer caso reportado en Uruguay.

\section{Materiales y métodos}

Se describe el caso de una perra American Staffordshire terrier de 6 años. El animal mostraba temblores, debilidad y ataxia como signos clínicos principales al momento de la primera visita al Centro Hospital Veterinario de la Facultad de Veterinaria de la Universidad de la República (FVET-UdelaR, Montevideo, Uruguay), desde ese momento, los signos neurológicos continuaron progresando lentamente durante los siguientes 6 meses, sin mejoría con ninguno de los tratamientos instaurados en la Unidad de Neurología de la FVET-UdelaR. Debido al mal pronóstico y por no existir posibilidad de recuperación del paciente, se recomendó a su propietario la eutanasia del animal, la que se realizó con su autorización, siguiendo el protocolo de eutanasia del Centro Hospital Veterinario de la FVET-UdelaR, usando para ello, una sobredosis intravenosa de tiopental sódico (protocolo aprobado por la Comisión de Ética en el Uso de Animales, CEUA, FVET-UdelaR). Inmediatamente después, se reali- zó la necropsia, centrándose la revisión en el sistema nervioso central, tomando diferentes muestras de órganos (encéfalo en su totalidad, médula espinal cervical, hígado, riñón, pulmón y bazo), cada muestra fue fijada en una solución de formol neutro al 10\%, para posteriormente realizar el examen histopatológico. Por otro lado, se realizaron estudios serológicos para medir los anticuerpos contra Neospora caninum y Toxoplasma gondii, los que resultaron positivos para ambos parásitos.

\section{Resultados}

Macroscópicamente, el cerebelo era más pequeño, presentando achatamiento del vermis cerebeloso, notándose porque no llegaba a llenar la fosa caudal que separa el cerebelo de los hemisferios cerebrales, mientras que las folias cerebelosas se notaban remarcadas y levemente adelgazadas, con los surcos interfoliares ensanchados y más profundos (Figura 1).

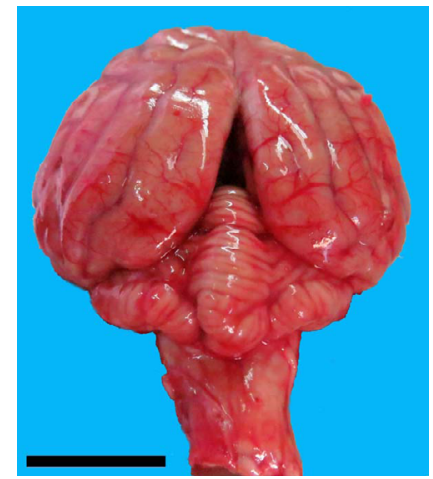

Figura 1. Abiotrofia cortical cerebelosa, perra, American Staffordshire terrier, 6 años. El cerebelo no llega a llenar la fosa caudal. Nótese la demarcación de los espacios interfoliares que aparecen como surcos más profundos por la retracción de las folias cerebelosas especialmente en el vermis cerebeloso (escala equivale a $2 \mathrm{~cm}$ ).

Al corte sagital del cerebelo, la retracción de las folias cerebelosas se hacía más evidente sobre la zona anterior del vermis cerebeloso, notándose una reducción proporcional de todos los lóbulos cerebelosos, midiendo en su eje mayor $22 \mathrm{~mm}$ (Figura 2).

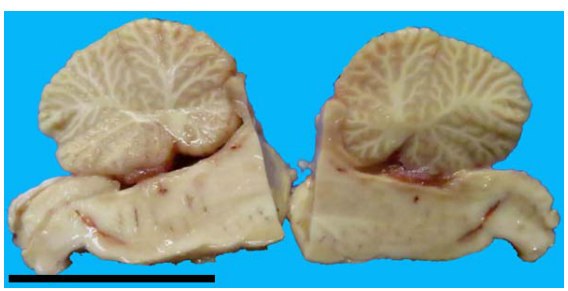

Figura 2. Abiotrofia cortical cerebelosa, perra, American Staffordshire terrier, 6 años. Corte sagital del cerebelo mostrando retracción del cerebelo, siendo más notoria en las regiones más anteriores en ambas hemi-secciones (escala equivale a $2 \mathrm{~cm}$ ).

No se encontraron otras anomalías en hemisferios cerebrales, diencéfalo, tronco cerebral y médula espinal, así como tampoco en los demás órganos estudiados durante el examen post-mortem del animal. Pese a ello, se comentan brevemente los principales hallazgos que se describieron en el informe de necropsia, en el examen exterior del cadáver se observaron en las caras 
externa de ambos muslos y en el mentón, áreas alopécicas de bordes irregulares con piodermatitis bacteriana de superficial a profunda, que eran compatibles con los antecedentes clínicos de atopia cutánea canina por la que el animal había sido tratado previamente. La topografía de la cavidad abdominal mostró hepatomegalia y esplenomegalia por congestión pasiva, mientras que en cavidad torácica se observaron algunas áreas de petequias, tanto la congestión pasiva del hígado y del bazo, así como las petequias de mediastino son hallazgos frecuentes en animales sometidos a eutanasia por sobredosis barbitúrica. Histológicamente, se observaron cambios histopstológicos característicos solamente en el cerebelo, el resto de los órganos estudiados, salvo la confirmación histológica de la congestión del hígado y el bazo, no presentaban hallazgos destacables.

La corteza cerebelosa mostró una estructura anormal, con adelgazamiento multifocal de la capa granulosa al disminuir los elementos celulares de la misma, también disminuyó notablemente el grosor de la capa molecular y se observó una importante pérdida de células de Purkinje (Figuras 3 y 4). En la capa molecular reducida, se encontró gran número de astrocitos hipertróficos (Figura 4).

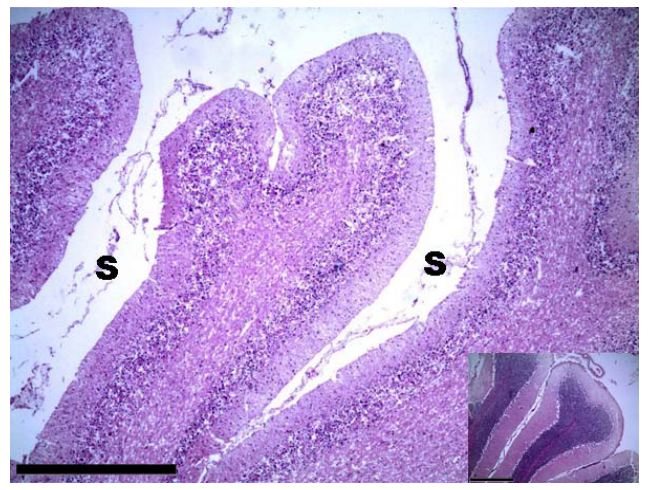

Figura 3. Abiotrofia cortical cerebelosa, perra, American Staffordshire terrier, 6 años. Adelgazamiento de una folia cerebelosa y surcos expandidos. $\mathrm{S}=$ surcos. El recuadro insertado muestra una folia control. HE. $\times 40$ (escalas de figura principal e inserto equivalen a $750 \mu \mathrm{m})$.

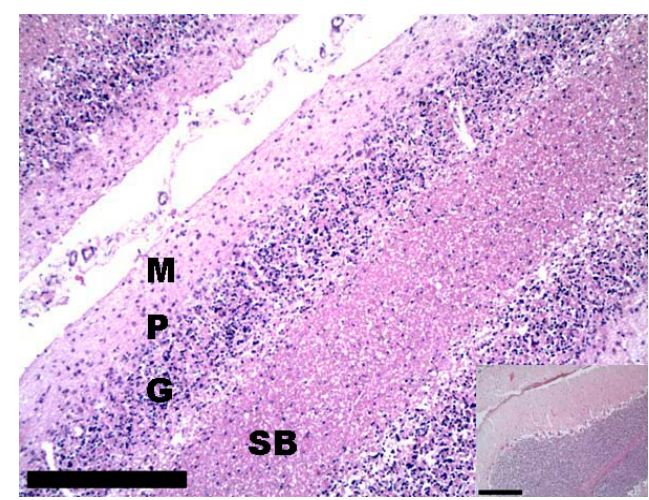

Figura 4. Abiotrofia cortical cerebelosa, perra, American Staffordshire terrier, 6 años. Adelgazamiento de las capas molecular y granulosa, con disminución del número de células de Purkinje. $\mathrm{M}=$ capa molecular, $\mathrm{P}=$ capa de células de Purkinje, $\mathrm{G}=$ capa granulosa, $\mathrm{SB}=$ sustancia blanca. El recuadro insertado muestra una folia control. HE. $\times 100$ (escalas de figura principal e inserto equivalen a $300 \mu \mathrm{m})$
En la capa granulosa, hubo una notoria disminución de los elementos celulares, llegando a observarse áreas con cambios patológicos más avanzados en los que la desaparición de la mayoría de las células granulosas era la imagen característica (Figura 5).

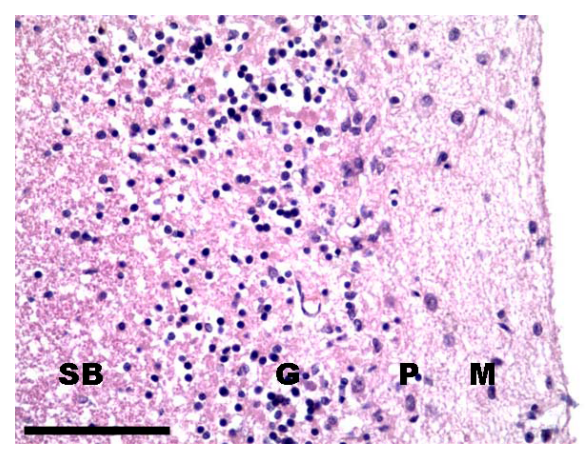

Figura 5. Abiotrofia cortical cerebelosa, perra, American Staffordshire terrier, 6 años. Perdida de células de Purkinje y disminución del número de células de la capa granulosa. $\mathrm{B}=$ sustancia blanca, $\mathrm{G}=$ capa granulosa, $\mathrm{P}=$ capa de células de Purkinje, $\mathrm{M}=$ capa molecular. HE. $\times 400$ (escala equivale a $75 \mu \mathrm{m})$.

En todas las capas de la corteza cerebelosa también se encontraron focos inflamatorios nodulares sobre las neuronas y sus prolongaciones. Por otro lado, también se detectó reacción astroglial, disminución de las neuronas de Golgi, y presencia de células de Purkinje ectópicas. En la capa de Purkinje, la pérdida celular progresó con la degeneración y la necrosis de las células de Purkinje, formándose pequeños focos vacuolares (cestas vacías, de su nombre en inglés "empty baskets") acompañados de la agregación de células gliales (Figura 6).

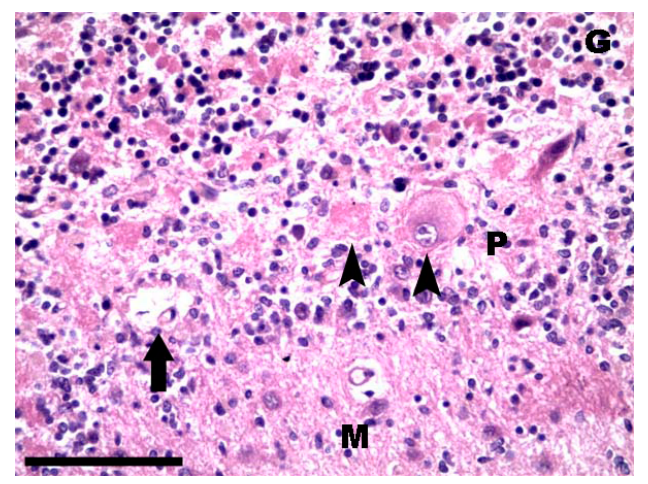

Figura 6. Abiotrofia cortical cerebelosa, perra, American Staffordshire terrier, 6 años. Células de Purkinje degeneradas y necróticas (cabezas de flecha), con presencia de canastas vacías acompañadas por células gliales (flecha). Aumento y engrosamientos de los astrocitos en la capa molecular. $\mathrm{G}=$ capa granulosa, $\mathrm{P}=$ capa de células de Purkinje, $\mathrm{M}=$ capa molecular. HE. $\times 400$ (escala equivale a $75 \mu \mathrm{m}$ ). 
En la sustancia blanca, se encontró vacuolización acompañada de gliosis. También se detectaron cambios similares de vacuolización con gliosis, en grupos de axones agrupados en nervios centrales y en la médula espinal (Figura 7).

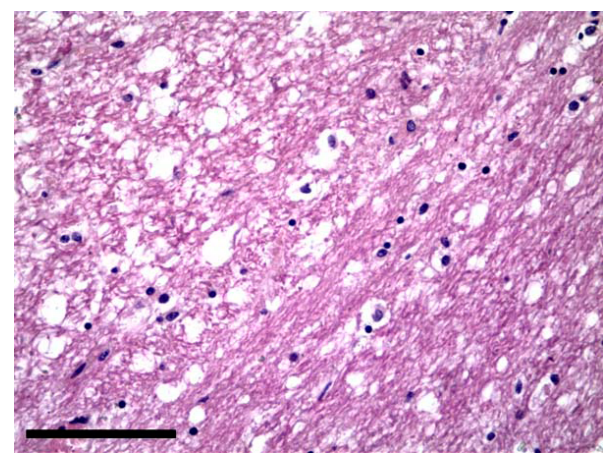

Figura 7. Abiotrofia cortical cerebelosa, perra, American Staffordshire terrier, 6 años. Vacuolización de la sustancia blanca por donde discurren las conexiones cerebelosas. HE. $\times 400$ (escala equivale a $75 \mu \mathrm{m}$ ).

No se detectaron en el cerebro algunas de las lesiones parasitarias características de Toxoplasma spp. y Neospora spp., incluyendo quistes de protozoarios, y tampoco se observaron las lesiones características de la infección con el virus del Distemper Canino.

\section{Discusión}

En el presente caso, los principales hallazgos histológicos fueron la disminución de las neuronas en la capa de células de Purkinje y en la capa granulosa, así como el adelgazamiento de la capa molecular con astrocitos hipertróficos. En los libros de texto, la abiotrofia cerebelosa se define como la degeneración prematura o acelerada de las neuronas cerebelosas completamente formadas (Cantile y Youssef, 2016). A partir de esta definición, queda claro que el presente caso se diagnostica como abiotrofia cortical cerebelosa. Por otro lado, varios cambios histopatológicos observados en este estudio fueron similares a los datos publicados para American Staffordshire terriers y otras razas diferentes (Thomas y Robertson, 1989; Chieffo et al., 1994; Sandy et al., 2002; Olby et al., 2004; Flegel et al., 2007; Jokinen et al., 2007; Henke et al., 2008; Urkasemsin et al., 2010; Forman et al., 2012; Kyostila et al., 2012; Urkasemsin et al., 2017). La relación entre la pérdida de células de Purkinje y la disminución de los elementos celulares en la capa granulosa se ha analizado a partir de las diferencias entre las razas y los tiempos de inicio, pero aún no se ha aclarado definitivamente su patogénesis (Olby et al., 2004; Shearman et al., 2011; Huska et al., 2013; Fenn et al., 2016). En este caso, los cambios en la capa granulosa y en las células de Purkinje fueron muy severos. Por lo tanto, no fue posible discutir la relación entre la disminución de las células de Purkinje y la pérdida de células en la capa granulosa. Además, la vacuolización y la gliosis también se observaron en otros nervios centrales conjuntamente con lo descrito para el cerebelo, y este tipo de cambios también fueron descritos de forma similar por otros autores (Higgins et al., 1998; Berry y Blas-Machado,
2003).

En cuanto al establecimiento del diagnóstico diferencial con otras enfermedades que afecten el sistema nervioso y particularmente el cerebelo de caninos domésticos, y teniendo en cuenta que el animal presentaba anticuerpos contra los protozoarios parásitos Neospora caninum y Toxoplasma gondii, el estudio histopatológico completo realizado en las muestras de sistema nervioso, incluyendo las diferentes regiones del cerebelo, no mostró en ningún caso los típicos quistes con formas intermedias de estos protozoarios o grupos de taquizoitos descritos en el sistema nervioso de perros afectados por estos parásitos (Dubey et al., 2002). Por otro lado, en casos neurológicos progresivos y severos, como el que se describe en este trabajo, no se podría descartar basándonos solamente en los signos clínicos, que los mismos fuesen ocasionados por una meningoencefalitis desmielinizante causada por el virus del Distemper Canino, para ello, recurriendo nuevamente al estudio histopatológico en el sistema nervioso, en el que se confirmó que las lesiones celulares se limitaban principalmente a la corteza cerebelosa, no encontrándose en ninguna de las muestras estudiadas las lesiones típicas de Distemper Canino, como ser la desmielinización severa, la neurodegeneración, la formación de manguitos perivasculares y/o la presencia de cuerpos de inclusión intranucleares e intracitoplásmicos, principalmente en astrocitos y neuronas de diferentes regiones del sistema nervioso.

La perra examinada en este estudio tenía 6 años de edad y se le realizó la necropsia a los 6 meses de iniciada la presentación de signos neurológicos. Es decir, la edad de inicio fue después de llegar a la adultez y el curso clínico fue de progresión lenta. Se ha informado que la aparición de signos clínicos en American Staffordshire terriers varía entre 3 y 13 años, y los signos clínicos progresan lentamente (Hanzlicek et al., 2003; Olby et al., 2004; Henke et al., 2008). El inicio de los signos clínicos de este trastorno y el curso posterior difieren según las razas de perros. En Beagles y Rhodesian ridgebacks se desarrollan rápidamente después del nacimiento (Chieffo et al., 1994; Forman et al., 2012) y se dice que para la mayoría de las razas se identifican semanas o meses después del nacimiento (Thomas y Robertson, 1989; Sandy et al., 2002; Flegel et al., 2007; Jokinen et al., 2007; Kyostila et al., 2012; Urkasemsin et al., 2017). Por otro lado, en los perros Old English sheepdogs, Gordon setters, Scottish terriers y American Staffordshire terriers se desarrolla después del año de nacimiento con una progresión más lenta (Hanzlícek et al., 2003; Speciale y de Lahunta, 2003; Olby et al., 2004; Henke et al., 2008; Urkasemsin et al., 2010; Agler et al., 2014, Urkasemsin et al., 2017). El tiempo de inicio y el curso clínico en este caso también fueron consistentes con los informados previamente en American Staffordshire terriers. Agler et al. (2014) sugieren que un defecto en RAB24, un gen asociado con la autofagia, estaría altamente asociado con la abiotrofia cerebelosa y podría contribuir a la ataxia hereditaria canina en perros Old English sheepdogs, mientras que Fenn et al. (2016) identificaron un empalme altamente asociado a mutación del sitio donante en SNX14, con un modo de herencia autosómico recesivo sospechado en los perros Vizsla húngaros. Por otro lado, 
se realizó un análisis de mapeo de todo el genoma en los perros Kelpie australianos de abiotrofia cerebelosa y no se detectaron los genes relacionados con la causa de la ataxia (Shearman et al., 2011). En nuestro caso, no hay registro de signos neurológicos similares a los descritos en esta perra en sus hermanos de camada, por lo que no fue posible discutir si hubo una relación familiar en este caso de abiotrofia cortical cerebelosa.

\section{Conclusiones}

Se examinó histológicamente el cerebro de una perra American Staffordshire terrier de 6 años que mostraba signos neurológicos de progresión lenta. En general, el cerebelo era más pequeño, aunque proporcionado, y las folias eran delgadas con surcos interfoliares expandidos. Histológicamente, los cambios característicos fueron la disminución en el número de elementos celulares en la capa granulosa, marcada pérdida de células de Purkinje y adelgazamiento de la capa molecular. A partir de estos cambios patológicos específicamente en el cerebelo, este caso fue diagnosticado como abiotrofia cortical cerebelosa canina de presentación tardía.

\section{Agradecimientos}

Gimena Feijoó es estudiante de MSc (Salud Animal) del Programa de Posgrados de la Facultad de Veterinaria (PPFVUdelaR, Uruguay). Kanji Yamasaki es docente contratado por el Programa de Contratación de Científicos Provenientes del Exterior (CSIC-UdelaR) y la FVET-UdelaR. José Manuel Verdes recibe financiación de CSIC-UdelaR, PEDECIBA y ANII (Uruguay). Luis Delucchi y José Manuel Verdes integran el SNI (Uruguay).

\section{Referencias bibliográficas}

Abitbol, M., Thibaud, J. L., Olby, N. J., Hitte, C., Puech, J. P., Maurer, M., Pilot-Storck, F., Hédan, B., Dréano, S., Brahimi, S., Delattre, D., André. C., Gray, F., Delisle, F., Caillaud, C., Bernex F., Panthier J.J., Aubin-Houzelstein G., Blot S., Tiret L. (2010). A canine Arylsulfatase G (ARSG) mutation leading to a sulfatase deficiency is associated with neuronal ceroid lipofuscinosis. Proc Natl Acad Sci USA, 107, 14775-14780.

Agler, C., Nielsen, D. M., Urkasemsin, G., Singleton, A., Tonomura, N., Sigurdsson, S., Tang, R., Linder, K., Arepalli, S., Hernández, D., Lindblad-Toh, K., van de Leemput, J., Motsinger-Reif, A., O’Brien, D. P., Bell, J., Harris, T., Steinberg, S., Olby, N. J. (2014). Canine hereditary ataxia in old english sheepdogs and gordon setters is associated with a defect in the autophagy gene encoding RAB24. PLoS Genet, 10, e1003991.

Berry, M. L., Blas-Machado, U. (2003). Cerebellar abiotrophy in a miniature schnauzer. Can Vet $J, 44,657-659$.

Cantile, C., Youssef, S. (2016). Nervous System. En: Maxie, M. G. (ed.). Jubb, Kennedy \& Palmer's Pathology of Domestic
Animals (6a. ed., pp. 250-406). London: Elsevier.

Carmichael, K. P., Miller, M., Rawlings, C. A., Fischer, A., Oliver, J. E., Miller, B. E. (1996). Clinical, hematologic, and biochemical features of a syndrome in Bernese Mountain Dogs characterized by hepatocerebellar degeneration. J Am Vet Med Assoc, 208, 1277-1279.

Chieffo, C., Stalis, I. H., Van Winkle, T.J., Haskins, M. E., Patterson, D. F. (1994). Cerebellar Purkinje's cell degeneration and coat color dilution in a family of Rhodesian Ridgeback dogs. J Vet Inter Med, 8, 112-116.

de Lahunta, A. (1990). Abiotrophy in domestic animals: a review. Can J Vet Res, 54, 65-76.

Dubey, J. P., Barr, B. C., Barta, J. R., Bjerkas, I., Björkman, C., Blagburn, B. L., Bowman, D. D., Buxton, D., Ellis, J. T., Gottstein, B., Hemphill, A., Hill, D. E., Howe, D. K., Jenkins, M. C., Kobayashi, Y., Koudela, B., Marsh, A. E., Mattsson, J. G., McAllister, M. M., Modry, D., Omata, Y., Sibley, L. D., Speer, C. A., Trees, A. J., Uggla, A., Upton, S. J., Williams, D. J. L., Lindsay, D. S. (2002). Redescription of Neospora caninum and its differentiation from related coccidian. Int J Parasitol, 32, 929-946.

Fenn, J., Boursnell, M., Hitti, R. J., Jenkins, C. A., Terry, R. L., Priestnall, S. L., Kenny, P. J., Mellersh, C. S., Forman, O. P. (2016). Genome sequencing reveals a splice donor site mutation in the SNX14 gene associated with novel cerebellar cortical degeneration in the Hungarian Vizsla dog breed. BMC Genet, 17, 123.

Flegel, T., Matiasek, K, Henke, D., Grevel, V. (2007). Cerebellar cortical degeneration with selective granule cell loss in Bavarian mountain dogs. J Small Anim Pract, 48, 462-465.

Forman, O. P., de Risio, L., Stewart, J., Mellersh, C. S., Beltran, E. (2012). Genome-wide mRNA sequencing of a single canine cerebellar cortical degeneration case leads to the identification of a disease associated SPTBN2 mutation. BMC Genet, 13,55 .

Gandini, G., Botteron, C., Brini, E., Fatzer, R., Diana, A., Jaggy, A. (2005). Cerebellar cortical degeneration in three English bulldogs: clinical and neuropathological findings. J Small Anim Pract, 46, 291-294.

Gill, J. M., Hewland, M. (1980). Cerebellar degeneration in the Border Collie. N Z Vet J, 28, 170.

Hanzlícek, D., Kathmann, I., Bley, T., Srenk, P., Botteron, C., Gaillard, C., Jaggy, A. (2003). Zerebelläre kortikale Abiotrophie beim American Staffordshire Terrier: Klinische und pathologische Beschreibung von drei Fällen. Schweiz Arch Tierheilkd, 145, 369-375.

Henke, D., Böttcher, P., Doherr, M. G., Oechtering, G., Flegel, 
T. (2008). Computer-assisted magnetic resonance imaging brain morphometry in American Staffordshire Terriers with cerebellar cortical degeneration. $J$ Vet Intern Med, 22, 969-975.

Higgins, R. J., LeCouteur, R. A., Kornegay, J. N., Coates, J. R. (1998). Late-onset progressive spinocerebellar degeneration in Brittany Spaniel dogs. Acta Neuropathol, 96, 97-101.

Huska, J., Gaitero, L., Snyman, H. N., Foster, R. A., Pumarola, M., Rodenas, S. (2013). Cerebellar granuloprival degeneration in an Australian kelpie and a Labrador retriever dog. Can Vet J, $54,55$.

Jokinen, T. S., Rusbridge, C., Steffen, F., Viitmaa, R., Syrjä, P., de Lahunta, A., Snellman, M., Cizinauskas S. (2007). Cerebellar cortical abiotrophy in Lagotto Romagnolo dogs. J Small Anim Pract, 48, 470-473.

Kent, M., Glass, E., de Lahunta, A. (2000). Cerebellar cortical abiotrophy in a beagle. J Small Anim Pract, 41, 321-323.

Kyostila, K., Cizinauskas, S., Seppala, E. H., Suhonen, E., Jeserevics, J., Sukura, A., Syrja, P., Lohi, H. (2012). A SEL1L mutation links a canine progressive early-onset cerebellar ataxia to the endoplasmic reticulum-associated protein degradation (ERAD) machinery. PLoS Genet, 8, e1002759.

Olby, N., Blot, S., Thibaud, J. L., Phillips, J., O’Brien, D. P., Burr, J., Berg, J., Brown, T., Breen, M. (2004). Cerebellar cortical degeneration in adult American Staffordshire Terriers. $J$ Vet Intern Med, 18, 201-208.

Perille, A. L., Baer, K., Joseph, R. J., Carillo, J. M., Averill, D. R. (1991). Postnatal cerebellar cortical degeneration in Labrador retriever puppies. Can Vet J, 32, 619-621.

Sandy, J. R., Slocombe, R. E., Mitten, R. W., Jedwab, D. (2002). Cerebellar abiotrophy in a family of Border Collie dogs. Vet Pathol, 39, 736-738.

Scott, E. Y., Woolard, K. D., Finno, C. J., Murray, J. D. (2018). Cerebellar abiotrophy across domestic species. The Cerebellum, 17, 372-379.

Shearman, J. R., Cook, R. W., McCowan, C., Fletcher, J. L., Taylor, R. M., Wilton, A. N. (2011). Mapping cerebellar abiotrophy in Australian Kelpies. Anim Genet, 42, 675-678.

Siso, S., Navarro, C., Hanzlicek, D., Vandevelde, M. (2004). Adult onset thalamocerebellar degeneration in dogs associated to neuronal storage of ceroid lipopigment. Acta Neuropathol, 108, 386-392.

Speciale, J., de Lahunta, A. (2003). Cerebellar degeneration in a mature Staffordshire Terrier. J Am Anim Hosp Assoc, 39, $459-462$.
Vet Med Assoc, 217, 1162-1165.

Thomas, J. B., Robertson, D. (1989). Hereditary cerebellar abiotrophy in Australian kelpie dogs. Aust Vet J, 66, 301-302.

Urkasemsin, G., Linder, K. E., Bell, J. S., de Lahunta, A., Olby, N. J. (2010). Hereditary cerebellar degeneration in Scottish Terriers. J Vet Intern Med, 24, 565-570.

Urkasemsin, G., Nielsen, D. M., Singleton, A., Arepalli, S., Hernández, D., Agler, C., Olby, N. J. (2017). Genetics of Hereditary Ataxia in Scottish Terriers. J Vet Intern Med, 31, 1132-1139.

van der Merwe, L. L, Lane, E. (2001). Diagnosis of cerebellar cortical degeneration in a Scottish terrier using magnetic resonance imaging. J Small Anim Prac, 42, 409-412.

van Tongeren, S. E, Van Vonderen, I. K., Van Nes, J. J., Van Den Ingh, T. S. (2000). Cerebellar cortical abiotrophy in two Portuguese Podenco littermates. Vet Q, 22, 172-174.

Yasuba, M., Okimoto, K., Iida, M., Itakura, C. (1988). Cerebellar cortical degeneration in Beagle dogs. Vet Pathol, 25, 315-317.

\section{Notas de contribución:}

1. Concepción y diseño del estudio, 2. Adquisición de datos, 3. Análisis de datos, 4. Discusión de los resultados, 5. Redacción del manuscrito, 6. Aprobación de la versión final del manuscrito. José Manuel Verdes ha contribuido en 1, 2, 3, 4, 5 y 6. Luis Delucchi ha contribuido en 2 y 4 . Gimena Feijóo ha contribuido en 2 y 4 . Fernando Fumagalli ha contribuido en 2. Carlos Rodríguez Serpa ha contribuido en 2. Kanji Yamasaki ha contribuido $1,2,3,4$ y 5 .

El editor Cecilia Cajarville aprobó ese artículo. 